\title{
A ampliação das políticas de saúde do homem na atenção básica prevenindo doenças: uma revisão bibliográfica
}

\author{
The expansion of men's health policies in primary care preventing diseases: a \\ bibliographic review
}

\section{La expansión de las políticas de salud masculina en atención primaria previniendo enfermedades: revisión bibliográfica}

Edmar Reis Thiengo ${ }^{1 *}$, Sirlene de Oliveira Mario Inacio', Lídia Silva do Nascimento Oliveira1, Juliana Silva Andrieta Andrade ${ }^{1}$, Sara Neves Ribeiro ${ }^{1}$, Griciane Romão de Souza Silva'.

\section{RESUMO}

Objetivo: Apresentar como as políticas de saúde do homem na atenção básica brasileira atuam na prevenção de doenças e como sua ampliação pode colaborar com a redução dos números de doenças em pessoas do sexo masculino. Revisão bibliográfica: As políticas de saúde do homem existem para prevenir o agravamento do quadro de doenças cujo a maior incidência é a população masculina, além de garantir maiores cuidados e prevenir o surgimento dessas doenças. A atenção básica brasileira compreende uma série de programas disponíveis para o primeiro contato do ser humano com as políticas de saúde pública. Cabe ressaltar, entretanto, que muitas políticas necessitam de um esforço maior dos profissionais da saúde para que o público alvo possa as aderir. Contudo, o grande empecilho que acaba não permitindo que essas políticas obtenham os dados desejados possui seu advento em uma cultura onde o homem não tem tempo para cuidados com a sua saúde. Considerações finais: Portanto, as políticas de saúde do homem carecem da ampliação das campanhas de adesão, além do fornecimento de maiores meios de acesso, como horários mais flexíveis que acompanham a agenda do trabalhador.

Palavras-chave: Saúde do homem, Política de saúde, Atenção primária à saúde.

\begin{abstract}
Objective: To present how the health policies of men in Brazilian primary care act in the prevention of diseases and how their expansion can collaborate with the reduction of the number of diseases in people of the male sex. Bibliographic review: Men's health policies exist to prevent the worsening of illnesses whose main incidence is the male population, in addition to ensuring greater care and preventing the onset of these diseases. Brazilian primary care comprises a series of programs available for the first contact of human beings with public health policies. It is worth mentioning, however, that many policies require a greater effort by health professionals so that the target audience can adhere to them. However, the great obstacle that ends up not allowing these policies to obtain the desired data has its advent in a culture where men do not have time to care for their health. Final considerations: Therefore, men's health policies lack the expansion of adherence campaigns, in addition to providing greater means of access, such as more flexible schedules that follow the worker's agenda.
\end{abstract}

Keywords: Men's health, Health policy, Primary health care.

\section{RESUMEN}

Objetivo: Presentar cómo las políticas de salud de los hombres en la atención primaria brasileña actúan en la prevención de enfermedades y cómo su expansión puede colaborar con la reducción del número de enfermedades en personas del sexo masculino. Revisión bibliográfica: Existen políticas de salud masculina para prevenir el agravamiento de enfermedades cuya principal incidencia es la población masculina, además de asegurar una mayor atención y prevenir la aparición de estas enfermedades. La atención primaria brasileña comprende una serie de programas disponibles para el primer contacto del ser humano con las políticas públicas de salud. Cabe mencionar, sin embargo, que muchas políticas requieren de un mayor esfuerzo por parte de los profesionales de la salud para que el público objetivo pueda adherirse a ellas. Sin embargo, el gran obstáculo que termina por no permitir que estas políticas obtengan los datos deseados tiene su advenimiento en una cultura donde los hombres no tienen tiempo para cuidar su salud. Consideraciones finales: Por lo tanto, las políticas de salud masculina carecen de la ampliación de las campañas de adherencia, además de brindar mayores vías de acceso, como horarios más flexibles que sigan la agenda del trabajador.

Palabras clave: Salud masculina, Política sanitaria, Atención primaria de salud.

${ }^{1}$ Faculdade Vale do Cricaré (FVC), São Mateus - ES. *E-mail: thiengo.thiengo@hotmail.com 


\section{INTRODUÇÃO}

Os primeiros estudos sobre a saúde do homem surgiram nos Estados Unidos no final dos anos 1970. Enfocando principalmente os problemas de saúde, alguns apontaram que, embora os homens sejam mais fortes do que as mulheres, eles estão em pior situação em termos de morbidade e mortalidade. Desde a década de 1990, essa abordagem tem se centrado nas peculiaridades do homem no processo de adoecimento a partir de uma perspectiva de gênero. Nos primeiros anos do século 21 , a saúde masculina passou a ser objeto de muitos estudos internacionais. Com base nisso, são apresentadas cinco hipóteses que explicam as diferenças entre homens e mulheres em termos de adoecimento e mortalidade: especificidade biológica e genética; diferenças sociais e étnicas e desigualdades sociais; relação entre comportamento e diferentes expectativas sociais; encontrar e usar serviços de saúde; disponibilidade de profissionais de saúde com orientação para a saúde masculina (SCHWARZ E, et al., 2012).

Tradicionalmente, os homens desconhecem suas particularidades e não estão entre as pessoas que costumam receber mais atendimento nos serviços de Atenção Básica à Saúde (ABS). O uso dos serviços de saúde pela mulher difere acentuadamente do dos homens, principalmente quando o foco é prevenção de doenças. Em geral, a procura de serviços médicos por homens é realizada em situações de extrema emergência e/ou em situações de urgência. Na construção de modelos de gênero, costuma-se representar o universo masculino como sexo forte ou fadado ao sofrimento. Tais objeções têm sido observadas na área da saúde. Nesse sentido, pode-se constatar que os homens consideram as necessidades de saúde ou cuidados primordiais, tarefas femininas (MOURA EC, et al., 2014).

Estudos epidemiológicos têm demonstrado que a expectativa de vida da população masculina é inferior à da população feminina mundial. Segundo a Organização Mundial da Saúde (OMS), a expectativa de vida média de homens e mulheres no mundo diferia em cinco anos em 2009: a expectativa de vida média das mulheres era de 71 anos e a dos homens era de 66 anos. Em termos de mortalidade, no Brasil, a diferença entre homens e mulheres entre 15 e 39 anos é significativamente maior: em 2010, os homens de 22 anos tinham 4,5 vezes mais chances de morrer do que as mulheres da mesma idade. Causas externas são a principal causa de morte de homens brasileiros nessa faixa etária (STORINO LP, et al., 2013).

Segundo dados do Ministério da Saúde (MS), os homens só procuram o Sistema Único de Saúde (SUS) por meio de atendimento especializado, o que é resultado do aumento da morbidade devido à demora no atendimento e ao alto custo do SUS. A desorganização das políticas de saúde desestimula o acesso da população masculina aos serviços de saúde e as campanhas públicas de saúde quase sempre não solucionam esse problema, com isso, os serviços de saúde também são considerados incapazes de atender às necessidades dos homens (DA SILVA PLN, et al., 2013).

Para Mendonça VS e Andrade AN (2010), as ações de promoção da saúde no cotidiano da própria Unidade Básica de Saúde (UBS) podem minimizar ou controlar os fatores que levam ao aumento da morbidade masculina. No entanto, como a saúde do homem não tem sido considerada em uma perspectiva mais abrangente, o envolvimento dos homens nas ações de saúde parece ser um desafio para o sistema público de saúde. Esta atenção mais específica permitirá que profissionais e os próprios homens tenham uma melhor compreensão das singularidades ou necessidades dos homens.

Outro fator que faz com que os homens não cumpram as medidas preventivas e de promoção da saúde é o estereótipo da masculinidade, que tem raízes na cultura e tem valores e crenças claros sobre o homem. Ou seja, o modelo hegemônico de masculinidade não reconhece fraqueza ou qualquer expressão que implique feminilidade. Já que doença ou enfermidade se referem à fraqueza e fragilidade, que é um aspecto típico da feminilidade, os comportamentos dos homens que não valorizam a saúde tornam-se habituais/naturais (MENDONÇA VS e ANDRADE NA, 2010).

Após uma problematização pautada na literatura existente, tem-se como objetivo demonstrar que as políticas de saúde do homem carecem da ampliação das campanhas de adesão, além do fornecimento de maiores meios de acesso, como horários mais flexíveis que acompanham a agenda do trabalhador. 


\section{REVISÃO BIBLIOGRÁFICA}

Desde o nascimento da humanidade, o processo histórico da cultura patriarcal promoveu a formação da masculinidade, que estabeleceu uma hierarquia entre homens e mulheres. Percebe-se que o homem tem a supremacia desde o início, considera-se invulnerável, é ensinado a não chorar e suprimir suas emoções, e considera a masculinidade como sinônimo de sexo. Com base em teorias arraigadas na história, a população masculina considera o cuidado à saúde um fenômeno único de masculinidade e ignora a importância da prevenção de doenças (CAVALCANTI JRD, et al., 2014).

No Brasil, o câncer de próstata é a segunda doença mais comum entre os homens. Em termos absolutos, é o sexto tipo mais comum no mundo e o tipo mais comum entre os homens, sendo responsável por cerca de $10 \%$ de todos os cânceres. No Brasil, a incidência desse câncer é de aproximadamente 6 vezes a dos países desenvolvidos. Nos últimos anos, a tecnologia revolucionou o conhecimento sobre o diagnóstico precoce, tratamento e cuidado do paciente com câncer de próstata. No entanto, a taxa de mortalidade no Brasil devido à doença está aumentando. Um problema central está relacionado a baixa adesão ao exame diagnóstico, principalmente no tocante ao toque retal, além dos fatores citados, o aspecto simbólico relacionado à sua intromissão também se destaca do ponto de vista físico e emocional, que está relacionado ao medo do próprio homem ao exame (PAIVA EP, et al., 2011).

Ainda para Cavalcanti JRD, et al. (2014), relacionado a esse fato está que a localização dos serviços de saúde pode induzir sentimentos de intimidação e distanciamento, levando ao desconhecimento das inúmeras possibilidades oferecidas pela Estratégia Saúde da Família (ESF), o que leva essa população a vulnerabilidade. No Brasil, desde a formulação oficial da Política Nacional de Saúde do Homem (PNAISH) em 27 de agosto de 2009, a saúde do homem foi gradativamente incluída na agenda da saúde pública. Entre os seguintes objetivos: qualificar os serviços médicos masculinos na perspectiva de garantir um sistema médico holístico, e qualificar a atenção primária para que não se limite à reabilitação, mas antes de tudo para garantir a promoção da saúde e a prevenção dos preveníveis.

Por muito tempo, diferentes departamentos de saúde e diferentes níveis de governo ignoraram a atenção à saúde masculina. No entanto, ao mesmo tempo, com a aprovação da Política Nacional de Atenção Integral à Saúde do Homem, tem havido cada vez mais discussões sobre o processo de saúde da clientela masculina. Por um lado, todos os aspectos desta política e normativa revelam os desafios que os gestores e profissionais de saúde devem enfrentar em particular, por outro, é urgente torná-la realidade a nível nacional, porque representa as necessidades e expectativas deste grupo de pessoas. Seu reconhecimento as questões de saúde são o principal problema de saúde pública (MOREIRA RLSF, et al., 2014).

No Brasil, o envelhecimento da população retrata a feminização como uma de suas marcas, e esse fenômeno ocorre porque os homens apresentam uma taxa de mortalidade maior do que as mulheres. A mortalidade em questão se divide em eixos causais de magnitude variável por faixa etária, e tem como principais causas as mortes externas e as doenças cardiovasculares, superiores ao câncer apenas na faixa etária acima de 50 anos. Diante da redução da demanda, percebeu-se que os homens não querem faltar ao trabalho, não estão preparados para resguardar a própria saúde e têm medo de adoecer e ficar dependente dos outros. Portanto, ao solicitarem medicamentos ou analgésicos, procuram atendimento médico na presença de doença instalada ou dor que atrapalhe seu desempenho no trabalho, por isso ignoram as consultas preventivas e valorizam as medidas curativas (PEREIRA LP e NERY AA, 2014).

Observa-se que os homens necessitam de políticas de saúde voltadas à promoção e prevenção, a fim de dar mais atenção às atividades de saúde próprias do homem e seu reconhecimento nas condições sociais, conscientizando-os de sua suscetibilidade a adquirir patologias suscetíveis ao homem. As propostas inclusivas dos homens nas ações de saúde são desafiadoras porque eles não percebem que cuidar e valorizar o corpo para fins de saúde é uma questão social. Devido à falta de conhecimento das campanhas de saúde pública dirigidas a homens e mulheres, os preconceitos existentes sobre os homens são óbvios porque raramente são discutidos e resolvidos (SILVA BTO, et al., 2013). 


\section{A Política Nacional de Atenção Integral à Saúde do Homem}

A Política Nacional de Atenção Integral à Saúde do Homem (PNAISH) foi criada pela portaria 1.944 de agosto de 2009, instituindo através do SUS a política nacional de saúde do homem. A portaria é composta de 9 artigos e delineia que as altas taxas de morbidade e mortalidade da população masculina representam um verdadeiro problema de saúde pública para o Brasil, justificando em muitos outros pontos a necessidade da legislação (BRASIL, 2009).

O decreto dispõe em seus artigos iniciais que:

"Art. 1 ㅇ Instituir, no âmbito do Sistema Único de Saúde (SUS), a Política Nacional de Atenção Integral à Saúde do Homem.

Parágrafo único. A Política de que trata o caput deste artigo visa promover a melhoria das condições de saúde da população masculina brasileira, contribuindo, de modo efetivo, para a redução da morbidade e da mortalidade dessa população, por meio do enfrentamento racional dos fatores de risco e mediante a facilitação ao acesso, às ações e aos serviços de assistência integral à saúde.

Art. 2o A Política Nacional de Atenção Integral à Saúde do Homem, de que trata o artigo $1^{\circ}$ desta Portaria, será regida pelos seguintes princípios: I - universalidade e equidade nas ações e serviços de saúde voltados para a população masculina, abrangendo a disponibilidade de insumos, equipamentos e materiais educativos; II humanização e qualificação da atenção à saúde do homem, com vistas à garantia, promoção e proteção dos direitos do homem, em conformidade com os preceitos éticos e suas peculiaridades socioculturais; III - co-responsabilidade quanto à saúde e à qualidade de vida da população masculina, implicando articulação com as diversas áreas do governo e com a sociedade; e IV -orientação à população masculina, aos familiares e à comunidade sobre a promoção, a prevenção, a proteção, o tratamento e a recuperação dos agravos e das enfermidades do homem" (BRASIL, 2009).

Um dos princípios enfatizados é a necessidade de mudar a percepção do homem sobre o cuidado de si e da saúde de sua família. Especificamente, o objetivo desta política é organizar, implementar, limitar e humanizar todo o território brasileiro no âmbito dos princípios que regem o SUS, e dar atenção plena à saúde masculina. Em geral, o objetivo é promover a melhoria da saúde masculina, contribuindo para a redução da morbimortalidade masculina, que é relativamente elevada em relação à feminina. Visa promover o acesso da população aos serviços integrais de saúde na atenção primária à saúde, para que a política tenha um caráter mais abrangente na atenção à saúde do homem (SEPARAVICH MA e CANESQUI AM, 2013).

Para financiar o PNAISH, em 2009 e 2011, os recursos foram repassados a todos os estados e Distrito Federal e a 26 municípios (capitais/médias/grandes cidades) no valor de $R \$ 75.000,00$ à vista. Em 2010, 54 municípios receberam os mesmos valores e, em 2011, 52 municípios receberam a parcela de $\mathrm{R} \$$ $55.000,00 /$ ano. Vale destacar que em 2012 foi publicada a portaria GM/MS 2.566 que previa o envio de $R \$$ $60.000,00$ aos municípios e foi revogada por não estar em consonância com os procedimentos de discussão e acordo tripartite. Dessa forma, o Ministério da Saúde destinaria um total de $R \$ 4.800 .000,00$ (quatro milhões e oitocentos mil reais) em custos de recursos aos municípios por ano (CONASS, 2013).

O Brasil dispersou a realização concreta da PNAISH em diversos serviços de assistência técnica em saúde pública e desconsidera a política histórica masculina do país no campo da saúde. A PNAISH é amplamente promovida pela Sociedade Brasileira de Urologia e foi criada em 2009 pelo Decreto $n$ o 1944 . Alguns dos princípios e diretrizes da PNAISH são baseados em dados epidemiológicos e fatores de risco relacionados à morbimortalidade (principalmente neoplasia) do câncer de próstata (CESARO BC, et al., 2018).

O objetivo geral dessa política é melhorar as condições de vida da população masculina, reduzir os fatores de risco e facilitar o acesso a ações e serviços médicos integrais, característicos da queda da morbimortalidade nessa população. Baseia-se nas diretrizes holísticas e organizacionais dos serviços públicos de saúde e acolhe os homens para que se sintam integrados. É implementada de forma hierárquica 
vinculada às estratégias de saúde da família, e sua implantação está vinculada às demais políticas e planos do Ministério da Saúde. Considerando que a temática do binômio "homens e saúde" ainda é pouco envolvida nos debates e discussões sobre saúde pública no Brasil, caracteriza-se pela insuficiente atenção à saúde da população masculina, podendo-se observar que um alto índice é causado por motivos evitáveis. As taxas de morbidade e mortalidade são altas, e a saúde do homem passar a ser um problema de saúde pública (ALVES BMS, et al., 2017).

No entanto, considerando que a maior taxa de doenças e mortalidade na população masculina vem sendo observada desde o século passado, e desde a Conferência Internacional de Desenvolvimento vem incentivando políticas públicas baseadas na perspectiva de gênero. No Cairo, em 1994, o que se questiona é a possível razão do desenho da PNAISH neste momento histórico. Para constituir o cenário ambíguo do discurso da PNAISH, utiliza-se a reconstrução histórica de seu processo de institucionalização. No entanto, é importante considerar que a história da PNAISH não está disponível em um único documento oficial. Ao contrário, está espalhado na literatura científica e em alguns textos que discutem sua construção, e até mesmo em textos midiáticos que dão às pessoas uma melhor compreensão do processo (MARTINS AM e MALAMUT BS, 2013).

\section{Empecilhos a adesão dos homens às Políticas Nacionais de Saúde}

Nas relações sociais diárias, as pessoas observam constantemente as características, atitudes e atributos comportamentais de homens e mulheres com base em suas personalidades. Este tipo de expectativas, expostas a partir de condições históricas, sociais e culturais específicas, sustentadas pelas diferenças percebidas entre homens e mulheres, acabam por constituir todo o sistema de relações de poder. De uma perspectiva teórica, os estudos de gênero afirmam as características sociais e culturais das distinções baseadas em gênero. Especificamente, masculinidade se refere a uma estrutura social que muda com mudanças nos fatores sociais, econômicos e de identidade com base na subjetividade da sociedade humana. Esses fatores incluem: geração, raça/etnia e sexo (BARROS CT, et al., 2018).

Cada grupo da sociedade estabelece comportamentos e hábitos específicos, resultando em diferentes estilos de vida. A diversidade desses grupos sociais é baseada em gênero, idade, região, tempo e fatores econômicos, políticos e culturais. Dessa forma, cada grupo expôs seu próprio conceito de saúde e doença a partir da compreensão da construção social e de sua interação com tantos outros fatores culturais. Em meados do século $X X$, as discussões sobre a masculinidade e o status dos homens em diferentes contextos sociais e culturais continuaram a se expandir. No final da década de 1970, surgiu a primeira leva de estudos sobre a saúde masculina, e foi somente na década de 1990 que se começou a explorar o tema da masculinidade no Brasil de uma perspectiva diferente e abrangente, avançando sobre a questão da vulnerabilidade masculina pela ausência de cuidados (MIRANDA SVC, et al., 2020).

Embora os homens sejam mais vulneráveis e morram mais cedo, raramente procuram os serviços médicos, especialmente os cuidados primários, porque pensam que não podem fazer nada. Isso ajuda a reduzir os cuidados e aumenta a probabilidade de adoecimento. Ressalta-se que os motivos que dificultam esse tipo de acesso são diversos, basicamente divididos em duas categorias: barreiras socioculturais e barreiras institucionais. As barreiras socioculturais estão relacionadas aos estereótipos de gênero, que acreditam que o homem é uma pessoa forte, enérgica e invulnerável. Essa ideia está relacionada à imagem da identidade de gênero e às questões culturais, pois há masculinidade que pode suprimir a demanda e os cuidados com a saúde (SIQUEIRA BPJ, et al., 2014).

Dentre as barreiras institucionais que impedem o serviço de saúde de encontrar homens, há uma que pode citar o horário de funcionamento da unidade, que coincide com a jornada de trabalho. Equipe de saúde composta principalmente por mulheres; demora no acesso ao atendimento; má recepção; preparação insuficiente dos profissionais; nenhum programa voltado para o público masculino; estruturas físicas que não permitem a privacidade dos participantes; instabilidade e baixa resolução dos serviços médicos, principalmente na atenção primária. A representação da sociedade reducionista de especialistas em saúde do sexo masculino fortaleceu a imagem desse tipo de homem, e eles não pensam que os homens sejam 
objetos médicos. Esse comportamento geralmente está relacionado ao despreparo desses profissionais e sua incapacidade de se adaptarem às novas necessidades de saúde específicas da população masculina, pois cada estilo de vida altera diferentes necessidades de saúde (SIQUEIRA BPJ, et al., 2014).

Em relação à maioria das doenças relacionadas às mulheres, os dados de mortalidade e imortalidade dos homens obscurecem a visão de que os homens têm menos doenças do que as mulheres. Isso mostra que a explicação desse fenômeno envolve também as questões de gênero, em que homens e mulheres desenvolvem diferentes comportamentos relacionados ao autocuidado sob a influência de diferentes fatores culturais. Além das questões de gênero, estudos têm demonstrado que outros fatores inerentes ao funcionamento dos serviços de saúde podem coexistir e impedir o acesso dos homens a esses serviços. Aponta-se alguns aspectos como os relacionados ao trabalho, à acessibilidade, à particularidade das equipes profissionais e à estrutura operacional desses serviços, além de outros fatores que afetam a redução da demanda dos homens por atendimento médico (ALVES RF, et al., 2011).

A relação de gênero é uma das dimensões organizacionais das relações sociais, sendo também a forma de saúde do homem e da mulher e elementos de intervenção no processo de tomada de decisão. Este conceito refere-se ao estabelecimento de diferenças e igualdade entre homens e mulheres, relações culturais e sociais de poder, a serem identificados como atributos masculinos e femininos nos mais diversos campos de atuação. Levando em consideração os princípios fundamentais da justiça para alcançar os demais gestores do sistema, bem como promover a atenção à saúde e o acesso aos serviços médicos, esse desafio está relacionado às questões de gênero, pois existem diferenças e diversidades entre homens e mulheres que devem ser consideradas (SOUSA DHAV, et al., 2015).

\section{CONSIDERAÇÕES FINAIS}

Entende-se a relação dos cuidados do homem com a saúde e a baixa adesão de ações preventivas, que além da busca pela integração do homem nas políticas públicas de saúde, há uma grande luta a ser travada no combate aos conceitos pré-existentes de masculinidade que afastam o homem das casas de saúde pública. Também deve-se conceber políticas públicas educacionais que visem reduzir a ideia de que o homem não deve inspirar cuidados para sua saúde, além de aumentar a disponibilidade dos programas da atenção básica de saúde em horários acessíveis a população masculina, que muitas das vezes está afastada pelo conflito dos horários disponíveis com a jornada de trabalho.

\section{REFERÊNCIAS}

1. ALVES BMS, et al. Atuação do enfermeiro da atenção básica diante das dificuldades para a implementação da política de saúde do homem. Rev enferm UFPE on line, 2017; 11(12): 5391-5401.

2. ALVES RF, et al. Gênero e saúde: o cuidar do homem em debate. Psicologia: teoria e prática, 2011; 13(3): $152-166$.

3. BARROS CT, et al."Mas se o homem cuidar da saúde fica meio que paradoxal ao trabalho": relação entre masculinidades e cuidado à saúde para homens jovens em formação profissional. Saúde Soc., 2018; 27(2): 423 -434.

4. BRASIL. 2009. In: PORTARIA № 1.944, de 27 deagostode 2009. Brasília: Ministério da Saúde. Disponível em: http://bvsms.saude.gov.br/bvs/saudelegis/gm/2009/prt1944 2708 2009.html. Acesso em: 1 de nov. 2020.

5. CAVALCANTI JRD, et al. Assistência Integral a Saúde do Homem: necessidades, obstáculos e estratégias de enfrentamento. Escola anna nery revistade Enfermagem, 2014; 18(4): 628-634.

6. CESARO BC, et al. Masculinidades inerentes à política brasileira de saúde do homem. Rev Panam Salud Publica, 2018; 42(1): 1-5.

7. CONASS. 2013. In: POLÍTICA Nacional de Atenção Integral à Saúde do Homem. Brasília: Progestores. Disponível em: https://www.conass.org.br/wp-content/uploads/2013/09/NT-41-2013-POLITICA-NACIONAL-SH.pdf. Acesso em 1 de nov. 2020.

8. DA SILVA PLN, et al. A Política de Atenção à Saúde do Homem no Brasil e os desafios da sua implantação: uma revisão integrativa. Enfermería Global, 2013; 32(1): 414-443.

9. MARTINS AM, MALAMUT BS. Análise do discurso da Política Nacional de Atenção Integral à Saúde do Homem. Saúde Soc. São Paulo, 2013; 22(2): 429-440.

10. MENDONÇA VS, ANDRADE AN. A Política Nacional de Saúde do Homem: necessidade ou ilusão?. Revista Psicologia Política, 2010; 10(20): 215-226.

11. MIRANDA SVC, et al. A visão do homem trabalhador rural norte-mineiro sobre o cuidado em saúde no contexto da atenção primária à saúde. Ciência \& Saúde Coletiva, 2020; 25(4):1519-1527. 
12. MOREIRA RLSF, et al. Dificuldades de inserção do homem na atenção básica a saúde: a fala dos enfermeiros. Escola anna nery revista de Enfermagem, 2014; 18(4): 615-621.

13. MOURA EC, et al. Atenção à saúde dos homensno âmbito da Estratégia Saúde da Família. Ciência \& Saúde Coletiva, $2014 ; 19(2): 429-438$.

14. PAIVA EP, et al. Barreiras em relação aos exames de rastreamento do câncer de próstata. Rev. Latino-Am. Enfermagem, 2011; 19(1): 1-8.

15. PEREIRA LP, NERY AA. Planejamento, gestão e ações à saúde do homem na estratégia de saúde da família. Escola anna nery revista de Enfermagem, 2014; 18(4): 635-643.

16. SCHWARZ E, et al. Política de saúde do homem. Rev Saúde Pública, 2012; 46(1): 108-116.

17. SEPARAVICH MA, CANESQUI AM. Saúde do homem e masculinidades na Política Nacional de Atenção Integral à Saúde do Homem: uma revisão bibliográfica. Saúde Soc., 2013; 22(2): 415-428.

18. SILVA BTO, et al. Promoção e prevenção da saúde do homem. Interfaces Científicas - Saúde e Ambiente, 2013; 2(1): 95-101.

19. SIQUEIRA BPJ, et al. Homens e cuidado à saúde nas representações sociais de profissionais de saúde. Escola anna nery revista de Enfermagem, 2014; 18(4): 690-696.

20. SOUSA DHAV, et al. Os Homens e as Práticas de cuidado em saúde. Gênero \& Direito, 2015; 4(1): 397-415.

21. STORINO LP, et al. Necessidades de saúde de homens na atenção básica: acolhimento e vínculo como potencializadores da integralidade. Escola Anna Nery, 2013; 17(4): 638-645. 